\title{
NUMERICAL RELATIVITY CM1-A AND CM1-B SESSIONS
}

\author{
J. NOVAK \\ Département d'Astrophysique Relativiste et de Cosmologie \\ UMR 8629 du CNRS - Observatoire de Meudon \\ 92195 Meudon Cedex FRANCE
}

\section{Introduction}

Numerical Relativity is concerned with solving the Einstein equations, as well as any field or matter equations on curved space-time, by means of computer calculations. The methods developed for this purpose up to now, as well as the addressed physical problems are getting more numerous every day. Thus, it is not surprising that among almost a hundred of parallel sessions of this ninth Marcel Grossman Meeting, the Numerical Relativity session has been one of those with the greatest number of contributed speakers. In all there have been 18 presentations, so that the session has been split into two parts (CM1-a and CM1-b). It covered many fields of general relativity and Computer Science however, this contribution tries to give a brief report. The interested reader should also look at the short and long contribution of each participant. The report is organized as follows. Tools and numerical techniques developed or used by speakers are reported in Sec. 2, Sec. 3 is then devoted to different possible formulations of Einstein equations. The study of critical collapses is addressed in Sec. 4 and numerical models of cosmic strings in Sec. 5. Finally, the numerical study of compact objects is presented, as sources of gravitational radiation in binary systems in Sec. 6, and as isolated stars in Sec. 7. Some concluding remarks are given in Sec. 8 .

\section{Tools and Techniques}

The talk of Andrzej Krasiński presented the system "Ortocartan", a tool for algebraic computing. New developments have been done after the previous published description 1 . The new programs are now able to calculate: the kinematic tensors of the flow and their evolution equations, the curvature tensor corresponding to given connection coefficients in any number of dimensions, the Lagrangian for a given metric by the Landau-Lifshitz prescription, the Euler-Lagrange equations from a given Lagrangian and to verify first integrals for sets of ordinary differential equations of second order or factor out a given factor in intermediate expressions. The programs are implemented in Codemist Standard Lisp computers that can be installed on any computer that uses either Linux or Windows 982. This talk was the only one on algebraic computing, the others dealt with solving the Einstein equations.

In that sense, the talk by Mark Miller focused on stability problems of numerical solutions and code validation with his diagnostic tools for Numerical Relativity. This scientific field involves the numerical integration of multiple sets of complicated partial differential equations. Nowadays, with the existence of multiple evolution

cm1: submitted to World Scientific on October 26, 2018 
schemes for numerous formulations of the Einstein Equations in $3+1$ form, code validation is an extremely important issue. Here, he introduced two powerful tools for code validation, aimed at validating both the consistency of the finite difference equations to the differential equations and the stability of the finite differencing scheme. These tools are, respectively, a residual evaluator of the Einstein equations in 4-dimensional covariant form and a von Neumann stability analysis of the full non-linear Einstein equations. Some of the formulations have been described in the session (see Sec. 3 below).

As far as numerical methods are concerned, the most popular for solving partial differential equations are certainly the finite-difference methods. Within this framework, High Resolution Shock-Capturing methods are very powerful when one wants to deal with strong shocks or gradients appearing particularly in hydrodynamic equations 3. These methods have, for example, been used by Harald Dimmelmeier and Florian Siebel in their works (see Sec. 7 and Sec. 3 below). Leo Brewin talked about an alternative method using a lattice of geodesic segments to provide the samples of the space-time metrict. A suitable lattice for a spherically symmetric space is a semi-infinite ladder. The curvature is, as with finite differences, obtained from a quadratic interpolation of the sampled metric. He reported on a combination of the ADM equations with the smooth lattice method producing long term stable evolution of a maximally sliced Schwarzschild black hole. Standard boundary condition have been applied, namely reflection symmetry at he throat and static conditions at the outer boundary. The results clearly show no signs of an instability out to $t=1000 \mathrm{M}$. Work is currently in progress in applying the smooth lattice method to Brill waves in 2D axisymmetric space-times.

A completely different type of numerical methods has been described by Philippe Grandclément who talked about multi-domain pseudo-spectral techniques 5. In particular, he showed that these methods are able to deal with equations appearing when one wishes to solve the 2-body problem in the context of general relativity. He showed their capability to solve elliptic equations, like scalar or vectorial Poisson equations, with non-compactly supported sources (i.e. sources extending to infinity). The use of a compactified external domain enables one to impose exact boundary conditions at infinity. Pseudo-spectral methods have also been used by Jörg Frauendiener for determining hyperboloidal initial data sets. This problem involves the determination of a suitable conformal factor which transforms from an initial data set in physical space-time to a hyperboloidal hypersurface in the ambient conformal manifold and, furthermore, a division by the conformal factor of certain fields which vanish on $\mathcal{I}$, the zero set of the conformal factor. The challenge is to numerically obtain a smooth quotient $\hat{E}$. These hyperboloidal initial data can be used to generate general relativistic space-times by evolution with the conformal field equations.

John Baker discussed the Speciality invariant, a geometrically invariant quantity which was first identified as a useful tool for an invariant estimate of the size of perturbations on a single black hole as in the context of the Lazarus project 0 . The invariant $\mathrm{S}$, has the value 1 for algebraically special space-times, such as for stationary black holes. For the case of head-on black hole collision initial data, it was demonstrated that the S-invariant can provide an effective estimate of when

cm1: submitted to World Scientific on October 26, 2018 
near-linear dynamics can be expected. Moving beyond perturbation theory, it was also shown that the S-invariant is also useful as an analytic tool for interpreting numerical black hole forming space-times. Its key attributes in this context are: 1 . It is fully coordinate invariant. 2. Unlike other invariants, it has no characteristic fall-off behavior. 3. Its value tends to differ from 1, indicating local algebraic generality, wherever non-trivial dynamics, such as radiation, are active in the space-time. This makes the S invariant an extremely useful summary of the geometric content in a numerical black hole space-time.

\section{Formulations}

This part of the session focused on the way of writing down Einstein equations and the relevant quantities. The aim is to study the different possible choices for the decomposition of these equations (the most popular being the ADM formalism), for the gauge, for the variables and the model for the matter. The choice will depend on the numerical stability and accuracy of the formulation, as well as the physical relevance of the model. For example, Philippe Grandclément and John Whelan modeled stationary space-times describing compact object binaries without gravitational radiation reaction. In this regime, the gravitational interaction is too strong to use weak-field approximation methods, but the time scale for decay of the orbits is still long compared to the orbital period. The formulation of this physical property has been however done in two different manners. Philippe Grandclément has used a conformally flat spatial metric (within ADM decomposition), whereas John Whelan maintained equilibrium by imposing a balance of incoming and outgoing radiation at large distances. The conformally flat spatial metric is also known as Wilson's 9 gauge and has also been used by Harald Dimmelmeier in his work.

Hyperbolic formulation of Einstein equations is an alternative approach to ADM formalism and has been studied by Hisa-aki Shinkai (with Gen Yoneda) in order to implement stable long time evolution in numerical relativity. He presented three kinds of hyperbolic systems (weakly/strongly/symmetric hyperbolic) in the Ashtekar formulation of general relativity for Lorentzian vacuum space-time, and showed how hyperbolicity helps for stable numerical evolutions. He also presented two sets of dynamical equations, which forces the space-time to evolve to the manifold that satisfies the constraint equations-gr the reality conditions or both as the attractor against these perturbative errors 1011 . Another alternative is the characteristic formalism, which has been used by Florian Siebel in his work (with José A. Font, Ewald Müller and Philippos Papadopoulos). This formalism is specifically tailored to study gravitational radiation and is based upon the characteristic initial value problem. the standard description of the "+" and " $\times$ " polarization modes of gravitational radiation is in terms of the real and imaginary parts of the Bondi news function at future null infinity. He presented recent tests and results of an axisymmetric fully general relativistic code including a perfect fluid matter field. A spherically symmetric version of the code is capable of reproducing long term stability of a relativistic polytrope model of a neutron star.

Finally, Jochen Peitz talked about studying non-ideal relativistic hydrodynamics. Modeling such dissipative processes requires non-equilibrium or irreversible

cm1: submitted to World Scientific on October 26, 2018 
thermodynamics12. Standard (or classical) irreversible thermodynamics show the serious problems that dissipative fluctuations propagate at an infinite speed. In addition short wavelength secular instabilities driven by dissipative processes exist and, finally, no well-posed initial value problem exists for rotating fluid configurations. A complete set of equations for dissipative relativistic hydrodynamics in $3+1$ representation has been provided. Furthermore, the case of a relativistic fluid flow onto black holes has been discussed within this framework.

\section{Critical Collapses}

This field of general relativity, describing the mathematical properties of the Einstein equations, has received most of its results from numerical calculations. Thus,

Jonathan Thornburg (with Sascha Husa, Christiane Lechner, Michael Pürrer and Peter Aichelburg) reported on a new numerical code for studying critical phenomena in the spherically symmetric self-gravitating nonlinear $\mathrm{SU}(2)$ sigma mode 13 , and the convergence tests to validate the code's accuracy. The numerical code is based on an outgoing-null-cone formulation of the Einstein-matter equations, specialized to spherical symmetry, with freely falling grid points. The diamond integral scheme of Gomez and Winicour for the matter equation have been used, and second order uniform convergence of the results - including the critical parameter $\mathrm{p}^{*}$ - with grid spacing has been shown. Due to the fact that investigations of critical phenomena in gravitational collapse have been primarily restricted to spherical symmetry because of its relative computational simplicity, Eric Hirschmann reported on the development of a code to evolve axisymmetric gravitational collapse and to investigate gravitational critical phenomena. As matter, he considered scalar electrodynamics.

\section{Cosmic Strings}

Vortex type defects, seen on a macroscopic scale as cosmic strings, are presumably the topological defects which are the most likely to exist. In his talk about numerical models of dynamic cosmic strings, Robert Sjödin showed how the field equations for a time dependent cylindrical cosmic string coupled to gravity have been reformulated in terms of geometrical variables defined on a 2+1-dimensional space-time by using the method of Geroch decomposition 14. Unlike the 4-dimensional space-time the reduced case is asymptotically flat. A numerical method for solving the field equations which involves conformally compactifying the space and including null infinity as part of the grid was described. It was shown that the code reproduces the results of a number of vacuum solutions with one or two degrees of freedom. The code is stable, accurate and exhibits clear second order convergence. The interaction between a Weber-Wheeler pulse of gravitational radiation and the string has been analyzed. The interaction causes the string to oscillate at frequencies proportional to the masses of the scalar and the vector fields of the string.

cm1: submitted to World Scientific on October 26, 2018 


\section{Gravitational Waves from Binary Systems}

Binary systems of compact stars (i.e. neutron stars or black holes) and their coalescence are the most powerful sources of gravitational radiation. Therefore, the study of such systems and of their evolution is crucial not only from an academic point of view, but also for the design of the future data-analysis of interferometric gravitational wave detectors. Although analytical work can be done (see, for example, the talks in Self-Gravitating Systems -SG1- session), numerical treatment is often unavoidable. The quasi-stationary approach of binary systems was presented in two talks: by Philippe Grandclément, using pseudo-spectral techniques he showed results about the final orbits of neutron stars15; by John Whelan who presented another stationary approximation to the space-time of a compact object binary. This approximation for black hole binary inspiral is an approximation for studying strong field effects while suppressing radiation reaction. In his talk, he used a nonlinear scalar field toy model to explain the underlying method of approximating binary motion by periodic orbits with radiation; to show how the fields in such a model are found by the solution of a boundary value problem and to demonstrate how a good approximation to the outgoing radiation can be found by finding fields with a balance of ingoing and outgoing radiation (a generalization of standing waves).

Another approach is the direct simulation of the merger, which have not yet been achieved for the black hole case. Still, Pedro Marronetti talked about the numerical determination of approximate initial solutions for the binary black hole numerical merger. He presented_approximate analytical solutions to the Hamiltonian and momentum constraint 16 equations, corresponding to systems composed of two black holes with arbitrary linear and angular momentum. The analytical nature of these initial data solutions makes them easier to implement in numerical evolutions than the traditional numerical approach of solving the elliptic equations derived from the Einstein constraints. Although in general the problem of setting up initial conditions for black hole binary simulations is complicated by the presence of singularities, he showed that the methods presented in his work provide initial data with $l_{1}$ and $l_{\infty}$ norms of violation of the constraint equations falling below those of the truncation error (residual error due to discretization) present in finite difference codes for the range of grid resolutions currently used. Thus, these data sets are suitable for use in evolution codes. Detailed results were presented for the case of a head-on collision of two equal-mass M black holes with specific angular momentum $0.5 \mathrm{M}$ at an initial separation of $10 \mathrm{M}$. A straightforward superposition method yields data adequate for resolutions of $h=M / 4$, and an "attenuated" superposition yields data usable to resolutions at least as fine as $h=M / 8$. In addition, the attenuated approximate data may be more tractable in a full (computational) exact solution to the initial value problem.

Calculating gravitational waveforms from inspiralling binaries is necessary by all means. Starting from this point of view, Manuela Campanelli presented a new program, Lazarus, intended to make the best use of all available technologies to provide an effective understanding of gravitational waves from inspiralling black hole binaries in time for imminent observations. Lazarus is based on a eclectic

cm1: submitted to World Scientific on October 26, 2018 
approach to black hole collisions which combines, into a unified effort, the best features of the post-Newtonian calculations $(\mathrm{PN})$ applicable in the slow adiabatic inspiralling phase up to the innermost stable circular orbit (Isco), full numerical relativity simulations (FN) of Einstein's equations able to handle the rapid dynamical plunge and merger phase, and close limit black hole treatments (CL) of a single perturbed black hole applicable in the final ring-down phase. A key element of this program is thus the numerical implementation of the interfaces FN-CL and FN-PN. The Lazarus collaboration have already completed a general approach to providing the FN-CL interface and expect to solve soon the problem of the PNFN interface. They have successfully applied this approach to head-on collisiond, which provided the first binary black hole waveforms in full 3D numerical relativity, and they are just now completing its extension to the more astrophysically relevant case of inspiralling binary black holes. For the the first time, this approach makes it possible to study the fundamentally nonlinear processes taking place during the final plunge phase of the collision of two well-separated black holes, allowing a more direct physical understanding of radiation waveforms, nonlinear spin-interactions, stability against initial configurations and indicating clearly when non-linear effects are important.

Still, there can be several different approaches for calculating the postNewtonian phase. Achamveedu Gopakumar talked about his work (with Anshu Gupta, Bala R. Iyer and Sai Iyer) on Padé approximants for truncated postNewtonian neutron star models. These Padé approximants, once constructed, showed to converge faster to the general relativistic solution than the truncated post-Newtonian ones 17. Detailed studies of equilibrium configurations of single neutron stars and their evolution indicate that in the stable branch the second order Padé model converges to the exact general relativistic model even better than the straightforward third order truncated (Taylor) PN model. Both the simpler one parameter Padé form and a more involved three parameter Padé form exhibit similar improvement over the Taylor models. The Padé models are thus quite robust, controlled and perform better than the simpler Taylor truncated models. It is better to use initial data obtained from a Padé approximant to the Taylor model than initial data from a straightforward post-Newtonian truncated model of the same order. This feature should be generic and extend to binary neutron stars and black holes, especially since a useful simplification in a two-body problem is via a reduction to an equivalent one-body problem, and prove useful in numerical studies of such systems in the future.

\section{Gravitational Collapse and Neutron Stars}

Gravitational collapse, which forms a neutron star or a black hole, has been studied by Harald Dimmelmeier (with José A. Font and Ewald Müller). He showed numerical simulations of matter flows evolving in the presence of strong (and dynamic) gravitational fields. In order to simplify the complexity of the gravitational field equations of general relativity, Wilson and coworkers mation scheme, where the 3-metric is chosen to be conformally flat, which reduces the Einstein equations to a set of 5 coupled elliptic equations. An axisymmetric

cm1: submitted to World Scientific on October 26, 2018 
general relativistic hydrodynamic code which is based upon this approach, and utilizes high-resolution shock-capturing schemes to solve the hydrodynamic equations has been presented, as well as a report on preliminary applications of the code to rotating neutron stars and supernova core collapse in axisymmetry. These results demonstrate the feasibility of the code to handle a variety of relativistic astrophysical situations. The code will be used in the near future to obtain information about gravitational radiation from rotating gravitational collapse.

Only two studies of the properties of neutron stars has been presented here, first by Florian Siebel, on the spherically symmetric polytrope model (see Sec. 3). Then, by Johannes Ruoff who talked about neutron star oscillations; with models including polytropic and realistic equations of state. He presented the derivation of the perturbation equations governing the oscillations of relativistic non-rotating neutron star models using the $(3+1)$-decomposition. He showed that the perturbation equations can always be written in terms of space-time variables only, regardless of any particular gauge. He demonstrated how to obtain the Regge-Wheeler gauge, by choosing appropriate shift and lapse. In addition, not only the 3-metric but also the extrinsic curvature of the initial slice have to satisfy certain conditions in order to preserve the Regge-Wheeler gauge throughout the evolution. He discussed varipus forms of the equations and show their relation to the formulation of Allen et al 18 New results have been presented for polytropic equations of state. For realistic equations of state the numerical evolutions exhibit an instability, which does not occur for polytropic equations of state. This instability is related to the behavior of the sound speed at the neutron drip point. As a remedy he showed a transformation of the radial coordinate $r$ inside the star, which removes this instability and yields stable evolutions for any chosen numerical resolution 19 .

\section{Closing Remarks}

The large number of presentations in this session is indicative of the robust level of interest that members of theoretical physics and astrophysics communities express in considering numerical approaches to general relativity. Many different fields have been addressed, from pure numerics and applied mathematics to neutron star properties and gravitational wave astronomy. It was then obvious that, considered the large (and various) number of parallel sessions of the conference, many very interesting contributions for numerical relativity also took place in other sessions. This was particularly true for the CM3 "Black Hole Collisions" chaired by Pablo Laguna and some more astrophysical sessions like " $r$-modes instabilities in Neutrons Stars" (APT7, chaired by John Friedman) or "binary Neutron Stars - Coalescing Neutron Stars" (APT3, chaired by Takashi Nakamura). In this latter, Masaru Shibata showed very important results on the merging of neutron stars coming from the first code in full general relativity 20. One can only regret that, with such a rich field of investigations as are Computer Methods in General Relativity, and with four parallel sessions, there have been no plenary lecture connected to it.

cm1: submitted to World Scientific on October 26, 2018 


\section{Dedication}

This session has been dedicated to Jean-Alain Marck who died on May $9^{\text {th }}, 2000$; he has been one of the leading specialists in Numerical Relativity and he should have chaired this session. We all miss him.

\section{References}

1. A. Krasiński, Gen. Rel. Grav. 25, 165 (1993).

2. A. Krasiński, Gen. Rel. Grav. 33, No 1 (2001). Also at gr-qc/0005081.

3. J. M므. Ibáñez et al, in the proceedings of the conference 'Godunov Methods: theory and "applications", Oxford, October 1999. Also at astro-ph/9911034.

4. L. Brewin, Class. Quant. Grav. 15, 2427 (1998).

5. Ph. Grandclément et al., gr-qc/0003072.

6. J. Frauendiener, gr-qc/9806103.

7. J. Baker et al., Class. Quant. Grav. 17, L149 (2000).

8. J. Baker and M. Campanelli, Phys. Rev. D 62, 127501 (2000).

9. J.R. Wilson and G.J. Mathews, Phys. Rev. Lett. 75, 4161 (1995)

10. H. Shinkai and G. Yoneda, Phys. Rev. D 60, 101502 (1999).

11. H. Shinkai and G. Yoneda, Class. Quant. Grav. 17, 4799 (2000). Also at gr-qc/0007034.

12. J. Peitz and S. Appl, Month. Not. Roy. Astron. Soc. 296, 231 (1998).

13. S. Husa et al., Phys. Rev. D 62, 104007 (2000).

14. K.R.P. Sjödin, U. Sperhake and J.A. Vickers, gr-qc/0002096.

15. S. Bonazzola, E. Gourgoulhon and J-A. Marck, Phys. Rev. Lett. 82, 892 (1999); and also E. Gourgoulhon et al. gr-qc/0007028

16. P. Marronetti et al., Phys. Rev. D 62, 024017 (2000).

17. A. Gupta et al., Phys. Rev. D 62, 044038 (2000).

18. G. Allen et al., Phys. Rev. D 58, 124012 (1998).

19. J. Ruoff, Phys. Rev. D , in press (2001), also at gr-qc/0003088.

20. M. Shibata and K. Uryu, Phys. Rev. D 61, 064001 (2000). 